\title{
Periods and clusters in Ising cellular automata
}

\author{
H J Herrmann†, H O Carmesin $\ddagger$ and D Stauffer $\$ \|$ \\ † SPhT, CEN Saclay, 91191 Gif-sur-Yvette, France \\ $\doteqdot$ Institut für Physik, Universität Mainz, 6500 Mainz, Federal Republic of Germany \\ § LHMP, Ecole Superieure de Physique et Chimie Industrielles, 10 rue Vauquelin, 75231 \\ Paris Cedex 05, France
}

Received 26 November 1986

\begin{abstract}
The dynamic properties of Q2R cellular automata for the Ising model are studied numerically on the square lattice, with particular emphasis on the periods observed for single spins, clusters of neighbouring spins and the whole lattice. We find some numerical indications for a 'cluster-period critical point' at a temperature below the Curie temperature of the Ising magnet.
\end{abstract}

\section{Introduction}

In cellular automata the change in each variable for every time step depends in a simple and predictable way on the current values of the variable in the neighbouring cells of the lattice [1-3]. Pomeau has suggested [4] that the so-called Q2R rule [5-7] should simulate the Ising magnet in a microcanonical ensemble. This method leads to the fastest known algorithm [5] ( $10^{9}$ steps per second and processor on a Cray) for Ising model simulations. Also other cellular automata have been investigated $[6,7]$ in connection with the Ising model. The present paper investigates these Q2R automata not from the point of computational speed but with respect to one of the crucial questions in cellular automata theory: does the system, or a part of it, settle into a periodic motion, without ever reaching a time-independent equilibrium?

In Q2R automata, each site $i$ of a lattice is occupied by a spin $S_{i}$ with the possible values -1 and +1 . One starts from a rather disordered configuration. At every time step, the sign of the spin is changed if and only if this spin has the same number of parallel and antiparallel neighbours. Thus this spin flip does not change the energy of the system, and therefore this type of simulation corresponds to a microcanonical ensemble with fixed energy, not to a canonical ensemble with fixed temperature. (Spins are not updated all at once but the square lattice is divided into two sublattices; first one, then the other, sublattice is updated. No magnetic field is taken into account.) A spontaneous magnetisation was shown [5] to appear for temperatures below the Curie temperature. However, for sufficiently low temperatures or energies, oscillations in the magnetisation were observed [5] which did not die down with increasing time and thus hampered its accurate determination. Trivially, after at most $2^{N}$ time steps a lattice with $N$ spins will have returned to its initial configuration since these cellular automata are completely deterministic and reversible, and since the lattice has only of Germany. 
$2^{N}$ different configurations. However, with nearly a million spins employed in [5], this effect cannot be the reason for the observed periods and would anyhow be negligible in the thermodynamic limit $N \rightarrow \omega$, even if only the smaller subset of configurations with a fixed energy is investigated. Instead we want to know if much smaller periods, not exponentially in lattice size, exist in our problem.

If such periods exist they imply that the system is not ergodic, i.e. even if one waits infinitely long one does not reach in the simulation a sufficient number of different states with the given energy. The reverse is not true: averages could be wrong even if the period is infinite for an infinite system.

We study here the more practical question of periods. If the whole lattice, or appreciable parts of it, are locked into a 'limit cycle' of finite period, the simulation may give serious deviations from the desired equilibrium results since then the oscillations in, for example, the magnetisation do not vanish if we wait longer. We thus check for periods of the lattice, or of parts of the lattice, and see in particular whether these periods diverge at a certain phase transition energy.

All simulations were made on square lattices of size $L \times L$, with $32 \leqslant L \leqslant 6400$; presumably these constitute by far the largest cellular automata lattices reported so far (much larger simulations on a Cray 2 were performed later [5]). The energy is normalised to be zero for a random spin distribution and to be -2 for all spins parallel; $E=-1.41$ is then the total energy at the Curie point. We first set all spins $S_{i}=-1$ and then select randomly some spins to be flipped, until we reach the desired energy. This configuration then is the starting point of the completely deterministic evaluation of the cellular automata; see [5] for computational details.

\section{Global periods}

First we check if the lattice as a whole has a finite period, which we call the global period $T$. For this purpose one monitors and stores the magnetisation as a function of time. If the magnetisation after $T$ time steps agrees with the initial one, we suspect a global period of $T$, store the present configuration, make $T$ additional time steps and compare the resulting configuration with the stored one. If they agree in all spins, the period of the lattice is indeed $T$, otherwise one searches further. If no lattice period is found it means that the true period is larger than the maximum observable period $\tau$ (roughly the observation time), in which case we call it 'infinite'. Since the model is completely reversible it is not possible that one first has to go through an extended transient regime before the periodic motion begins. If a period is found, the initial configuration is already part of it.

Figure 1 shows as a function of energy $E$ the probability $W_{x}$ for an 'infinite' global period $T$, with lattice length $L$ varying over more than two orders of magnitude, and $\tau \sim 10^{3}$. The larger the lattice, the closer to unity is that probability. Presumably in the limit $L \rightarrow \infty$ this probability is unity for all $E$, i.e. infinitely large lattices have infinitely large periods. The average period $\langle T\rangle$, averaged over those samples for which such a lattice period was found, is shown in figure 2 and diverges at about that energy where the probability curve of figure 1 reaches unity. All data for $L \geqslant 128$ were obtained with a vectorised FORTRAN program [5] on a Cray-XMP computer.

At first sight these results would suggest that for sufficiently large lattices the simulation is not locked into finite periods and covers a large enough phase space to give good averages. However, such a conclusion would be dangerous since it could 


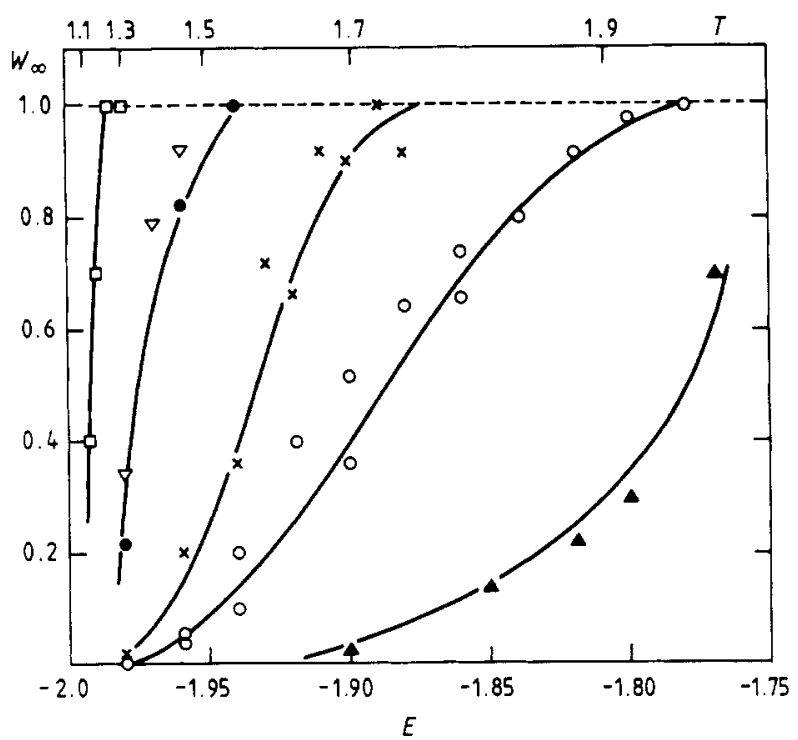

Figure 1. Probability $W_{\infty}$ that the $L \times L$ lattice has an infinite global period as a function of energy $E$ for various lattice lengths $L(\square, 6400 ; \nabla, 1280 ; \bigcirc, 640 ; \times, 256 ; 0,128 ; \boldsymbol{\Delta}, 32)$. The lines are guides to the eye.

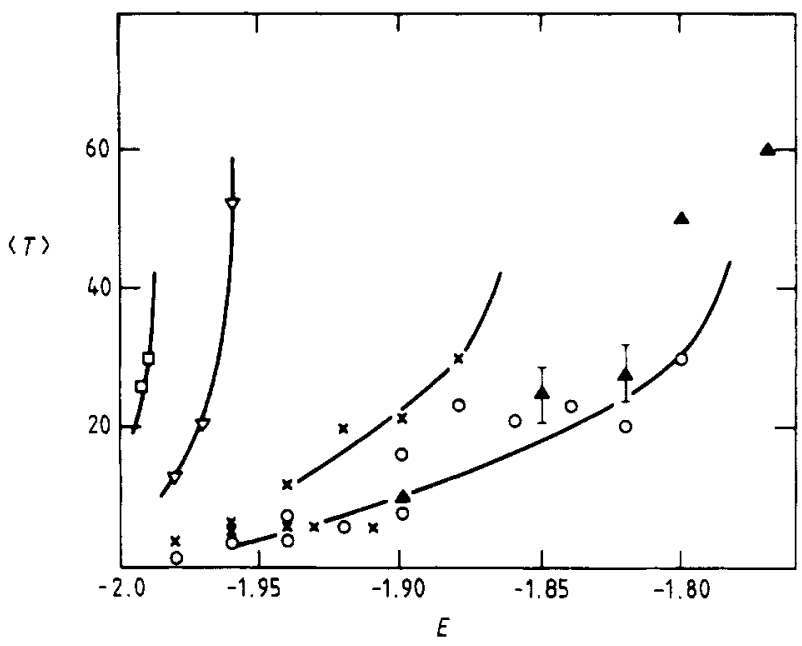

Figure 2. Average global period $\langle T)$ of $L \times L$ lattice as a function of energy $E(\square, L=6400$; $\nabla, L=640 ; \times, L=256 ; O, L=128 ; \boldsymbol{\Delta}, L=32$ ). The lines are guides to the eye.

be that one part of the lattice has period 5, another period 13, a third period 51, etc. Then the global period of the whole lattice would be the lowest common multiple of the various periods of all the different parts, which would be at least 3315 and thus 'infinite' in the above example. Figures 1 and 2 thus do not prove that in a sufficiently large lattice no sites have small local periods. Therefore the next section looks at such local periods which may be of more fundamental interest but are more memory consuming in their evaluation.

(For $L \leqslant 20$, the average global period increases roughly exponentially with the number of spins at high temperatures; at low temperatures that increase is weaker.) 


\section{Clusters}

Figure 3 illustrates the 'cluster' structure of our cellular automata. We show for each lattice site the period after which the sequence of \pm 1 values is repeated again and again. We see lots of sites with period 1, i.e. sites which do not change at all within the observation time. As a cluster we define sets of neighbouring sites having a finite period larger than unity, and thus shown in our figure 3 by $2,3,4, \ldots$. We see that we have lots of clusters and that within a cluster different periods are possible. In figure 4 we show as an example a cluster of period 7. When two sites of different period touch each other, one of the periods is an integer multiple of the other, as required by the definition of these automata. We also see that in contrast to percolation clusters, and also in contrast to those observed for Kauffman's cellular automata [9], our clusters are not very ramified but prefer rectangular shapes. Perhaps they are not fractal. One can easily see $[7,8]$ that clusters must stay within the smallest rectangle that can be drawn around a cluster of +1 spins in a surrounding of -1 spins (or vice versa). Our definition of clusters requires at least that if the observation time was long enough each finite cluster is surrounded by sites with period 1, as is confirmed by these pictures. Sites with periods larger than the maximum observable period $\tau$ will be denoted by a zero.

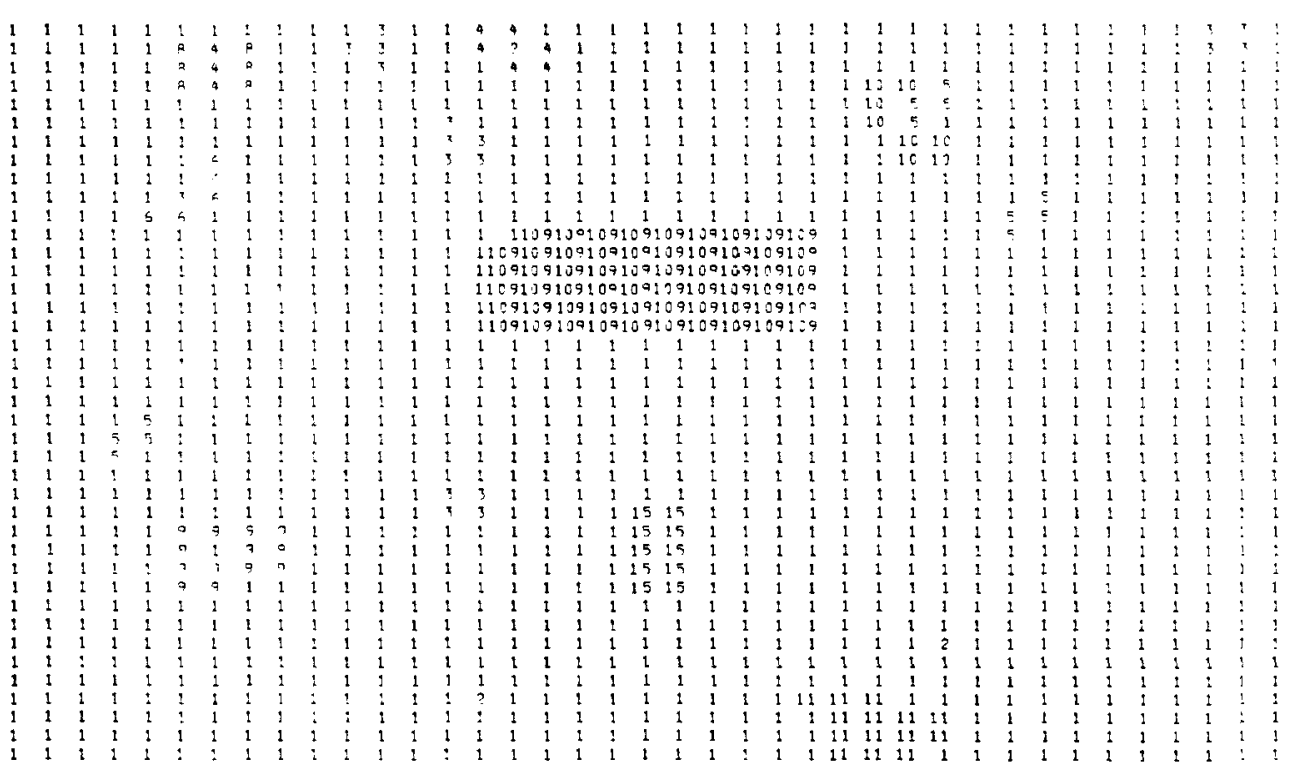

Figure 3. Picture of single-site periods and clusters for energy $E=-1.75$ near the effective 'cluster period' phase transition.

We see in our finite systems a drastic difference between high and low energies (high and low temperatures). For low energies all lattice sites have an observable and usually short period, whereas for high energies most of them have 'infinite' period and will be marked by a zero. This network of aperiodic sites percolates through the whole lattice for high energies but does not exclude the existence of finite clusters with finite periods, located within the holes of the infinite network of infinite-period sites.

To find the periods of a single site we store its total history, not just a few 'snapshots' as for the global periods. For a given trial period we then check whether the spin 


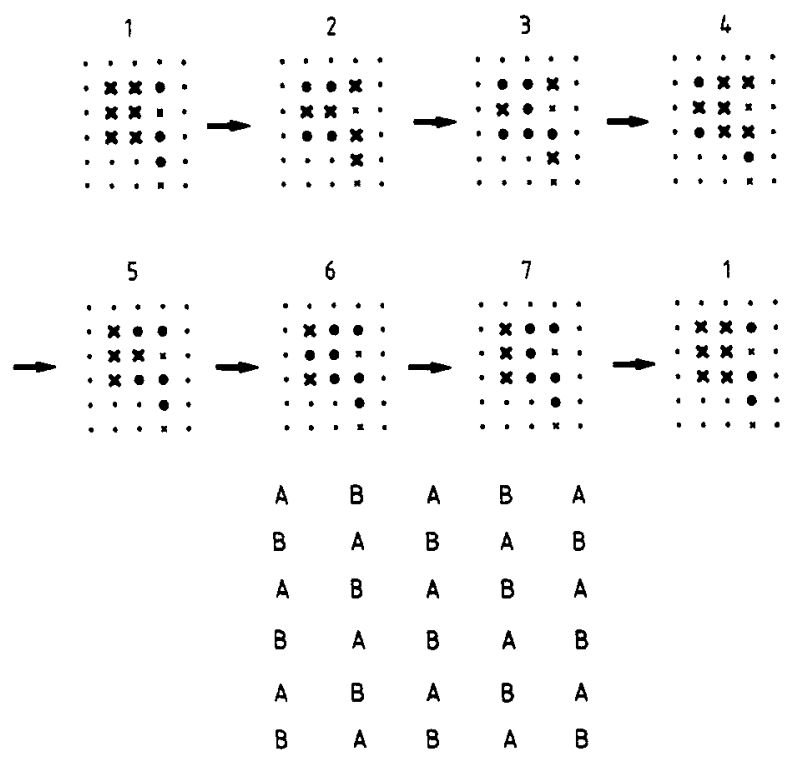

Figure 4. Example for the time development of a cluster with period seven.

orientations at times differing by this trial period are the same throughout the simulation; if not, we increase the trial period by one time unit and try again. This analysis, to be repeated for each site separately, is therefore quite time and memory consuming. To limit the computer memory requirements we stored for each time one site in one bit only. This work was therefore not done on a vector computer and was thus restricted to lattice sizes up to $64 \times 64$, in contrast to the much larger lattices for the global periods. The data are based on at least 13 simulations for the same set of parameters.

Figure 5 shows the observed single-site period and the fraction of sites having an infinite period, as a function of energy. The first quantity seems to diverge, the second

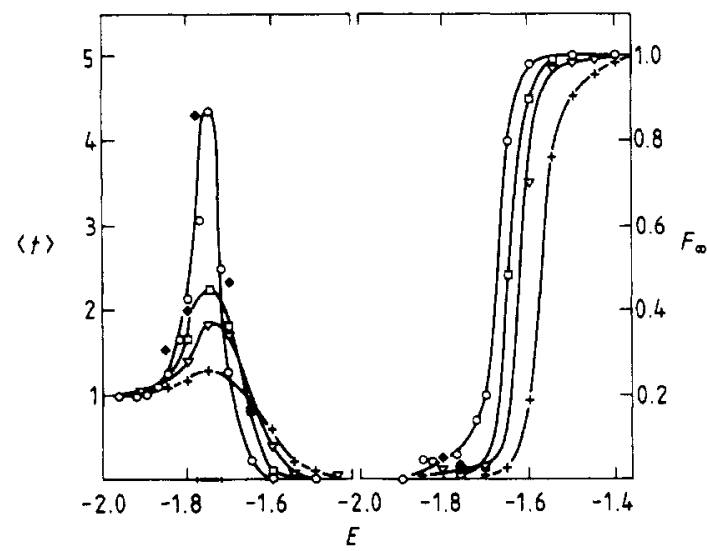

Figure 5. Average single-site period ( $t\rangle$ (left-hand side) and fraction $F_{\infty}$ of sites having 'infinite' periods (right-hand side) plotted against energy $E$ shown for different observation times (maximum observable period $\tau$ ). (,$+ \tau=32 ; \nabla, \tau=80 ; \square, \tau=160 ; 0, \diamond, \tau=320$ ). Data are for $64 \times 64$ lattices except $32 \times 32$ for the full diamonds. In the average for $\langle i\rangle$ the sites with period larger than $\tau$ are treated as having period zero. 
quantity seems to vanish, at about the same threshold energy $E_{\mathrm{cp}} \simeq-1.75$. (In our averages, sites without known period are treated as sites with period zero.) Our quantities in figure 5 are defined purely on the basis of single-spin variables, without any reference to clusters. For a smaller lattice of $32 \times 32$ instead of $64 \times 64$ sites, the systematic effects from the finite system size seem to be smaller than the statistical fluctuations. More important are the systematic trends with the observation time $\tau$, as seen in figure 5. They have similar influences as the finite-size effects at usual critical points: the height of the peak in the average period substantially increases as a function of $\tau$, presumably as a power law. Unfortunately our data are not accurate enough to extract a critical exponent. On the right-hand side of figure 5 we also see a weak but systematic shift of the effective threshold towards smaller energies, with increasing $\tau$. It cannot indeed be excluded that the threshold will move to zero temperature if the observation time and system size both go to infinity.

For energies larger than this 'cluster period energy' $E_{\mathrm{cp}} \simeq-1.75$ we still found sites with finite periods; however, when we increased the observation time $\tau$, the fraction $1-F_{\infty}$ of such sites seems to approach zero, as shown in figure 6. Apparently for $E$ near -1.5 one has sites which oscillate periodically over hundreds of time steps but finally become aperiodic.

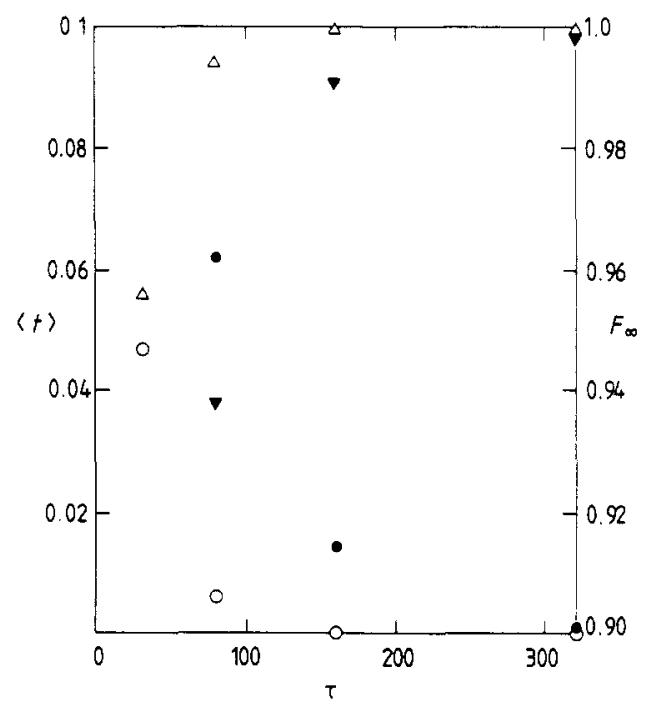

Figure 6. Variation with maximum observable period $\tau$ of the fraction $F_{\infty}$ of sites with 'infinite' period $(\triangle, E=-1.45, \nabla, E=-1.55)$, and of the average single-site period $\langle t\rangle$ (O, $E=-1.45 ; O, E=-1.55$ ). (In this average, infinite periods are set equal to zero.)

Returning now to the clusters, we characterise each cluster separately by three quantities.

(i) Its period, which we define as the largest period observed for any site within that cluster.

(ii) Its mass, which is the number of sites belonging to that cluster.

(iii) Its length, which is the 'chemical distance' between top and bottom of the cluster, i.e. the minimum number of nearest-neighbour jumps one has to make to go from one end of the cluster to the other. (This length is defined by the 'burning' algorithm [10].) 
Figure 7 shows the number of clusters observed at the energy -1.75 as a function of period, mass and length. The three curves look qualitatively quite the same, except that period one is missing since an isolated site (cluster of mass unity) must have a period of 2, always flipping back and forth. It would be nice to classify clusters with respect to two or three quantities simultaneously [11]. However, to get such information reliably would require much better statistics.

More quantitatively, figure 8 shows the second moment $\chi_{t}$ of the cluster period distribution as a function of energy. (Our average is done as for the 'mean cluster size' in percolation theory.) Again this quantity seems to diverge at $E \simeq-1.75$ for lattice size $64 \times 64$. For $E \leqslant-1.75$ in all samples the sites with infinite period formed a cluster spanning the whole lattice in at least one direction; for $E \geqslant-1.65$ they never did.

It seems therefore that the dynamics of Q2R cellular automata have a cluster period' threshold $E_{\mathrm{cp}}$ (near $E=-1.75$ for our $64 \times 64$ lattices) below the Curie point energy $E_{c}=-1.41$. At this cluster period threshold the single-site periods and the cluster periods diverge, whereas the number of sites with infinite period vanish there; thus these sites stop there to percolate through the lattice. The precise localisation of this threshold is difficult since there is a systematic trend by which the threshold moves for not too large observation times $\tau$ (see the right-hand side of figure 5). Larger $\tau$ and larger system sizes $L$ are needed to settle the question if this threshold takes place at finite temperatures or at zero temperature $\left(E_{\mathrm{cp}}=-2\right)$ and to determine the critical exponents at this threshold.

For temperatures above the Curie temperatures all spins of the lattice were moved after sufficiently long time; the time needed to flip all spins at least once seems to diverge, or to increase drastically, at the Curie point, whereas below the Curie point we could not flip all spins.

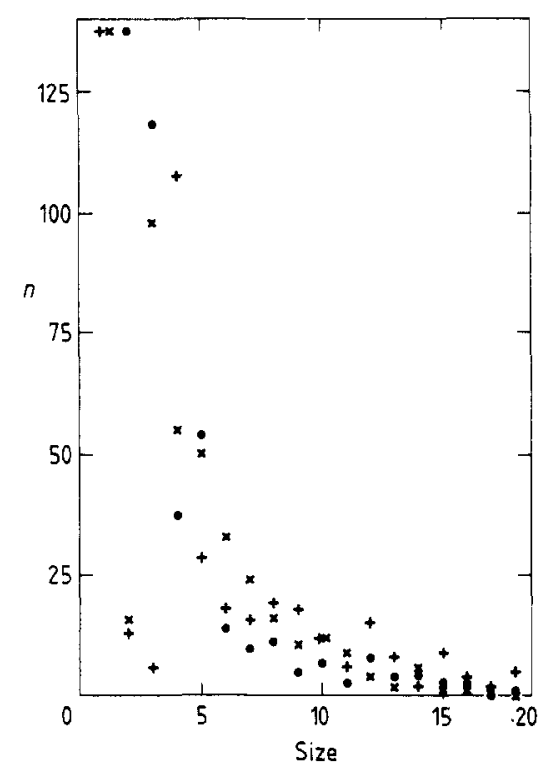

Figure 7. Cluster numbers observed in 13 runs of $64 \times 64$ lattice at $E=-1.75$, as classified by the maximum single-site period $(O)$, the number of sites ('mass') $(+)$ and the chemical distance ('length') of the cluster $(x)$ for $\tau=320$. 


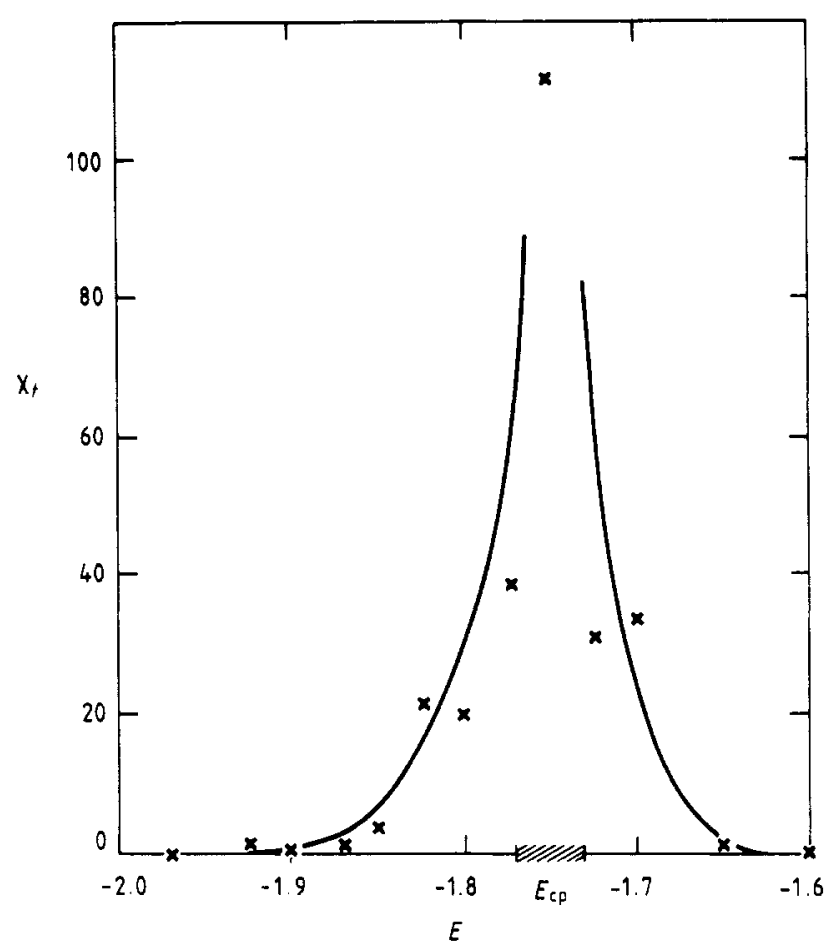

Figure 8. Second moment of the cluster period distribution as a function of energy. The lines are guides to the eye ( $L=64, \tau=320,13$ samples).

\section{Conclusions}

In this paper we have seen numerically, for rather small lattices, that the Q2R cellular automata have three phases: one is above the Curie point $E_{\mathrm{c}}=-1.41$ where the spontaneous magnetisation vanishes (as confirmed in [5]) and where all spins finally move. In the second one, below $E_{\mathrm{c}}$ and above $E_{\mathrm{cp}}$, the cluster sizes and periods can be arbitrarily large ('infinite' cluster); and finally in the third phase below $E_{\mathrm{cp}} \simeq-1.75$ there is no percolating cluster. We suggest calling $E_{\mathrm{cp}}$ the 'cluster period' critical point.

Since the spontaneous magnetisation is known to be entirely smooth for all finite temperatures below the Curie energy $E_{c}, E_{\mathrm{cp}}$ seems to have no influence whatsoever on the spontaneous magnetisation of the Ising model. This situation of two conceptually different critical points is somewhat similar to that observed in the three-dimensional Ising model [12]: the mean size of 'clusters' defined as groups of neighbouring parallel spins diverges at a temperature about $4 \%$ below the real Curie temperature. Some differences to that situation should, however, be pointed out: the present investigation was made for two, not for three, dimensions, and for two dimensions one does not have this $4 \%$ shift. Moreover, these static clusters of [12] do not influence at all the magnetisation whereas in our case the existence of finite periods lets the magnetisation oscillate and prevents it from reaching its true equilibrium value. In this sense our cluster period critical point indicates perhaps the limit energy (or temperature) below which these Ising cellular automata should not be utilised to simulate the normal Ising model. (It still needs to be checked whether it gives all averages correctly for higher temperatures [13].) Of course, in the sense of cellular automata the present model [6] 
has its own right of existence even at low temperatures. Finally we mention that our clusters look more compact than those of Ising magnets, percolation [14], or Kauffman cellular automata [9].

The critical point $E_{\mathrm{cp}}$ that we found might well shift, even to $T=0$, if the system size $L$ tends to infinity. In a related problem of bootstrap percolation it has been shown [15] that the threshold goes to zero as $1 / \log (L)$. Some arguments by Aizenman for our model indicate that $2+E_{c \mathrm{p}}(L)$ might vary as $1 / \log (L)$ for sufficiently large systems. Such a slow asymptotic shift of $E_{\mathrm{cp}}$ is very difficult to verify numerically for $L \leqslant 64$ and the shifts observed in figures 1 and 2 for much larger $L$ can be interpreted differently, as explained in $\S 2$. Since our data neither confirm nor contradict this hypothesis [15] of a logarithmic shift, we leave it here as an open problem.

From the practical point of view, our finite periods make simulations of Ising magnets with this algorithm unreliable for $E<E_{\mathrm{cp}}(L)$; however, even for higher energies and infinite periods one might get wrong averages or at least violate the ergodicity principle. The spontaneous magnetisation calculated in [5] indicates that at not too low temperatures any deviations between the true equilibrium values and the simulated averages are at most very small. We have not yet tested, e.g. with the methods of [16], how many different large basins of attraction exist for our dynamic process, or how results depend on the way to produce the initial configuration. An ergodic-non-ergodic transition of the type produced in [17] is therefore possible. The deterministic nature of our cellular automata prevents us from leaving a 'limit cycle' of finite period, even if we wait very long. If it should turn out that certain parts of the phase space are not accessible but are needed in order to obtain good averages, one might introduce a certain degree of randomness into the algorithm by randomly flipping some spins, after long time intervals. We report separately [18] on the dynamical effects caused by isolated perturbations [9] of the system.

Finally, we mention that the recent Creutz algorithm [19] of the microcanonical ensemble is a generalisation of the Q2R rule employed here. Creutz associates with each site a kinetic energy expressed by $m$ computer bits and works mainly with $m=2$. In the limit $m=0$ one recovers $Q 2 R$.

\section{Acknowledgments}

We thank Y Pomeau, B Derrida, M Aizenman and G Vichniac for helpful discussions.

\section{References}

[1] Wolfram S 1983 Rev. Mod. Phys. 55601

[2] Bienenstock E, Fogelman Soulié F and Weisbuch G (ed) 1986 Disordered Systems and Biological Organisation (Berlin: Springer)

[3] Farmer D, Toffoli T and Wolfram S (ed) 1984 Physica D 10 1-247

[4] Pomeau Y 1984 J. Phys. A: Math. Gen. 17 L415

[5] Herrmann H J 1986 J. Stat. Phys. 45145

Zabolitzky J G and Herrmann H J 1987 J. Comput. Phys. to be published

[6] Vichniac G Y 1984 Physica D 1096

[7] Viñals J and Gunton J D 1986 J. Phys. A: Math. Gen. 19 L933

[8] Vichniac G Y 1986 Disordered Systems and Biological Organisation ed E Bienenstock, F Fogelman Soulié and G Weisbuch (Berlin: Springer) p 17 
[9] Fogelman Soulié F 1985 Thesis Grenoble University

Derrida B and Stauffer D 1986 Europhys. Lett. 2739

Weisbuch G and Stauffer D 1987 J. Physique 4811

[10] Herrmann H J, Hong D C and Stanley H E 1984 J. Phys. A: Math. Gen. 17 L261

[11] Havlin H, Trus B, Weiss G H and Ben Avraham D 1985 J. Phys. A: Math. Gen. 18 L247

[12] Müller-Krumbhaar H 1974 Phys. Lett. 50A 27

Kertész J, Stauffer D and Coniglio A 1983 Percolation Structures and Processes ed G Deutscher, R Zallen and $J$ Adler (Bristol: Adam Hilger) p 121

[13] Lang W M and Stauffer D 1987 J. Phys. A: Math. Gen. 205413

[14] Stauffer D 1985 Introduction to Percolation Theory (London: Taylor and Francis)

[15] Aizenman M 1986 Private communication

[16] Derrida B and Flyvbjerg H 1986 J. Phys. A: Math. Gen. 19 L1003

[17] Isola S, Livi R and Ruffo S 1986 Preprint

[18] Stanley H E, Stauffer D, Herrmann H J and Kertész J 1986 Preprint

[19] Creutz M 1986 Ann. Phys., NY 16762 